BNL-79690-2007-CP

\title{
Using FFAGs in the Creation of Neutrino Beams
}

\author{
J. Scott Berg
}

To appear in the proceedings of the Ninth International Workshop on Neutrino Factories, Superbeams, and Betabeams, August 6-11, 2007, Okayama University, Okayama, Japan

November 2007

Physics Department/BIdg. 901A

\author{
Brookhaven National Laboratory \\ P.O. Box 5000 \\ Upton, NY 11973-5000 \\ www.bnl.gov
}

Notice: This manuscript has been authored by employees of Brookhaven Science Associates, LLC under Contract No. DE-AC02-98CH10886 with the U.S. Department of Energy. The publisher, by accepting the manuscript for publication, acknowledges that the United States Government retains a non-exclusive, paid-up, irrevocable, world-wide license to publish or reproduce the published form of this manuscript, or allow others to do so, for United States Government purposes. 


\section{DISCLAIMER}

This report was prepared as an account of work sponsored by an agency of the United States Government. Neither the United States Government nor any agency thereof, nor any of their employees, nor any of their contractors, subcontractors, or their employees, makes any warranty, express or implied, or assumes any legal liability or responsibility for the accuracy, completeness, or any third party's use or the results of such use of any information, apparatus, product, or process disclosed, or represents that its use would not infringe privately owned rights. Reference herein to any specific commercial product, process, or service by trade name, trademark, manufacturer, or otherwise, does not necessarily constitute or imply its endorsement, recommendation, or favoring by the United States Government or any agency thereof or its contractors or subcontractors. The views and opinions of authors expressed herein do not necessarily state or reflect those of the United States Government or any agency thereof. 


\title{
Using FFAGs in the Creation of Neutrino Beams
}

\author{
J. Scott Berg \\ Brookhaven National Laboratory, P. O. Box 5000, Upton, NY 11973-5000
}

\begin{abstract}
A number of accelerator-based facilities have been proposed for the creation of neutrino beams: superbeams, neutrino factories, and beta beams. Fixed field alternating gradient accelerators (FFAGs) have potential uses in all of these facilities. Superbeams and neutrino factories require high power proton drivers for the production of pions; FFAGs can beneficial for accelerating protons for those machines. FFAGs can reduce the cost of accelerating muons in a neutrino factory because they enable the muons to make many passes through the RF cavities and still accelerate rapidly. FFAGs have potential uses in production of radioactive ions for a beta beam facility, since radioactive ions that decay into high energy neutrinos in their rest frame may potentially be produced in a ring, and the large energy acceptance of an FFAG may be useful for maximizing beam lifetime in such a ring. Finally, FFAGs have been contemplated for use in ionization cooling rings for neutrino factories, since the equilibrium distribution in ionization cooling has a large energy spread for which an FFAG's large energy acceptance is needed, and FFAGs may make it feasible to inject and extract from such a ring.
\end{abstract}

Keywords: FFAG, fixed field alternating gradient accelerator, neutrino factory, superbeam, beta beam PACS: $29.20 .-\mathrm{c}, 29.38 .-\mathrm{c}$

\section{INTRODUCTION TO FFAGS}

Fixed field alternating gradient accelerators (FFAGs) are accelerator rings that have a very large energy acceptance, generally at least a factor of 2 in momentum. They use alternating gradient focusing to minimize the horizontal magnet apertures (in contrast to cyclotrons). FFAGs are of interest when rapid acceleration is desired, and the rate at which magnet fields can be varied would limit the acceleration rate in a synchrotron.

The primary problem that must be faced in any FFAG is the variation of the time of flight with energy. The RF frequency must change during the acceleration cycle, or acceleration must be completed before the particles slip in RF phase to the point that they are no longer accelerated. This time of flight variation generally becomes a limitation on the design of FFAGs: the ability to rapidly vary the RF frequency competes against the desire to have high voltages for rapid acceleration, or the number of turns in a machine is limited by the amount by which the RF phase changes in a turn.

Cyclotrons address this problem by making the time of flight independent of energy. This comes at the cost of large horizontal aperture and difficulty in reaching high energies, the latter because large fields and gradients are needed at relativistic energies to maintain isochronism.

\section{Types of FFAGs}

The original type of FFAG [1] is known today as a scaling FFAG. The tune of a scaling FFAG is independent of energy, thus the machine will avoid beam loss due to problematic resonances.

In recent years, other types of FFAGs have been proposed. Linear non-scaling FFAGs [2, 3] use linear magnets and consist of short, identical cells. The perfect machine therefore has only to be concerned by nonlinear resonances of a single cell, which should be weak due to the use of only linear magnets. Imperfections lead to additional resonances, but they are weak, and if one accelerates rapidly enough, they have minimal effect. They have smaller horizontal apertures than scaling FFAGs. At relativistic energies, this makes their time of flight variation with energy significantly less than scaling FFAGs.

Nonlinear non-scaling FFAGs [4] use nonlinear magnets to improve some of the properties of linear nonscaling FFAGs. In particular, they could reduce the time of flight variation from energy even more, reduce the tune variation with energy, or have a larger energy range. This potentially comes at the cost of dynamic aperture, since the assumption that nonlinear resonances are weak is no longer correct. These machines are considered because their properties may be better than those of a scaling FFAG for the same problem.

\section{PROTON DRIVERS}

High-power proton drivers are needed for both superbeams and neutrino factories. The primary requirement for either facility is the proton driver power (protons per second times the final proton energy). In addition, a neutrino factory needs a sequence of short bunches on the target (1 ns, but $3 \mathrm{~ns}$ is tolerable [5]). These bunches cannot come too often, since it takes a some amount of 
time to accelerate the beam and re-fill the RF cavities between pulses. Furthermore, when a liquid jet target is used, it takes some time (around $20 \mathrm{~ms}$ ) to replenish the jet after it is destroyed by the proton bunch. In a neutrino factory, the optimal efficiency of muon production for a given proton power is obtained with a proton energy in the range of 5-30 GeV [6].

Other types of machines can encounter difficulties in achieving these specifications. Isochronous cyclotrons have difficulty achieving energies much beyond $1 \mathrm{GeV}$, since the field gradients must get large to maintain isochronism. Synchrotrons can have difficulty ramping their magnets at a sufficient rate. A linac is likely to be expensive, and would need additional rings to produce the bunch structure needed for a neutrino factory. These difficulties motivate the consideration of FFAGs.

Space charge forces are a significant effect in any high-power proton driver. These forces increase when the number of particles in the beam increases or when the bunch length decreases. Higher repetition rates and higher energies are therefore preferred so as to have fewer protons per bunch for a given proton driver power. The space charge force also decreases as the beam energy increases. It is thus easier to produce the short bunches required for a neutrino factory at higher energies. However, the machine cost increases with the amount of acceleration, and it also takes longer to accelerate to higher energies, reducing the repetition rate.

Space charge forces cause particular problems for linear non-scaling FFAGs [7]. Linear non-scaling FFAGs have a tune which varies with energy, and therefore crosses nonlinear resonances. Space charge forces give nonlinearity, and there can be emittance growth and beam loss when the tune crosses the corresponding resonances. Thus, one must either accelerate very rapidly in a linear non-scaling FFAG, or one must use an FFAG design (scaling or nonlinear non-scaling) which has little or no tune variation with energy. Accelerating extremely rapidly can be a problem since at the low frequencies required to be able to vary the cavity frequency, the required voltages for sufficiently rapid acceleration may be difficult or expensive to achieve.

Constant-tune FFAGs are thus preferred (over variable-tune FFAGs) for a high-power proton driver. To determine if there is any hope for using a linear non-scaling FFAG, one should

- Perform simulations to determine the acceleration rate required in a linear non-scaling FFAG to keep emittance growth at acceptably low levels.

- Continue research programs on high-gradient RF systems that allow rapid frequency variation, to determine achievable acceleration rates.

An experiment, called MINHA, has been proposed to study space charge effects in linear non-scaling FFAGs [8]. It would accelerate low-energy electrons $(218-817 \mathrm{keV})$ with sufficient current $\left(5.5 \times 10^{11}\right.$ electrons) to have significant space charge tune shift.

A nonlinear non-scaling FFAG with nearly constant tunes has been proposed as part of a neutrino factory proton driver [4]. It appears to have a smaller aperture compared to scaling FFAGs for the same purpose. It also has a smaller momentum compaction factor, which allows the transition energy to be above the maximum ring energy, as well as enabling adiabatic bunch compression to the length required for a neutrino factory without an excessive RF voltage requirement. Similar machines have had some difficulties with dynamic aperture, and thus tracking studies must be performed on this design to ascertain if it has sufficient dynamic aperture.

Achieving higher acceleration rates would be easier if one could use high-frequency RF systems. However, such systems will not allow the variation of the frequency that is required because of the time of flight variation with energy. However, if one designs the machine so that on any given turn, the time of flight is an integer number of RF periods, but that number of periods changes from one turn to the next as the energy increases, then one can successfully accelerate [9, 10]. The primary challenges of these systems are making the energy gain depend on the current particle energy, which is required due to the nonlinear relationship between time of flight and energy, and constructing a high-frequency cavity with a sufficiently wide horizontal aperture.

\section{LARGE ENERGY SPREAD BEAMS}

Due to their large energy acceptance, FFAGs are useful in applications which do not require an energy increase in the beam, but do need to transport beams with a large energy spread. Scaling FFAGs which operate in this mode are currently under construction in Japan [11, 12, $13,14]$. In the production of neutrino beams, one must often deal with charged particle beams with large energy spreads, and thus FFAGs are of potential interest there.

\section{Radioactive Ion Source for Beta Beams}

A storage ring has been proposed to produce radioactive isotopes that decay into high-energy neutrinos in the ion rest frame. This would allow a beta-beam to accelerate to significantly lower energy per nucleon than for other isotopes. The ring would have an ion beam passing through a wedge-shaped gas target [15].

According to the paper, the equilibrium energy spread would be very small in such a ring. However, there seems 
to be some disagreement as to whether the calculation there was correct [16]: if the calculation is not correct, the energy spread will grow without bounds if there is not mixing between all three planes of motion. If the energy spread indeed does grow without bounds, an FFAG would be an ideal machine to store the beam. The larger the machine's energy acceptance, the longer one can store the beam before its energy spread increase results in excessive beam loss $[13,17]$. If one does have mixing between all three planes of motion, then one still has potentially large equilibrium energy spreads (from the numbers in [16], full momentum acceptances could be $\pm 3.5 \%$ or higher) where an FFAG might be needed.

\section{Ionization Cooling}

Ionization cooling is useful for reducing the transverse and longitudinal emittance of muon beams [18, 19, 20]. It seems to be optimal to have at least a small amount of ionization cooling in a neutrino factory, since that increases the number of muons that are transmitted to the storage ring [21]. As in the case of the production of radioactive ions, ionization cooling either results in a continuous increase in the energy spread, or, for the case where the machine achieves longitudinal cooling, there is an equilibrium energy spread which must be relatively large because of energy straggling.

Due to the large energy acceptance needed, some authors have considered FFAGs for an ionization cooling system [22, 23, 24]. It is not clear whether these are really well suited for ionization cooling, since at the required energies (momentum near $200 \mathrm{MeV} / c$ ), one can generally focus more strongly with solenoids. They may be useful in early stages, however, where equilibrium emittances can be relatively large.

\section{MUON ACCELERATION}

The application that originally motivated the development of the linear non-scaling FFAG was muon acceleration $[2,3]$ for a muon collider, which is very similar to muon acceleration for a neutrino factory. Since muons decay, it is essential that they be accelerated extremely rapidly: average gradients should be well above $1 \mathrm{MV} / \mathrm{m}$. This is significantly faster than the acceleration in proton drivers. To achieve these gradients, it is essential to use high- $Q \mathrm{RF}$, meaning that one cannot adjust the RF frequency to match the variation of time of flight with energy in an FFAG. Furthermore, one has no time to vary the fields in the magnets in a ring (as in a synchrotron).

The transverse and longitudinal emittances of the muon beam are very large (typical normalized emit- tances are are $7 \mathrm{~mm}$ transverse and $0.025 \mathrm{eV} \mathrm{s}$ longitudinal [25]). The acceleration systems thus need large physical and dynamic apertures. In particular, this limits the maximum acceleration frequency to around $200 \mathrm{MHz}$, at least at lower energies.

The most expensive components of the acceleration system are the RF subsystems. It is thus essential to reduce the amount of RF required to accelerate, meaning that one must make as many passes as possible through the RF cavities. Recirculating linear accelerators (RLAs), which consist of one or more linacs and arcs (a different arc for each energy) that transport the beam from one linac to the next, are limited to around 4 or 5 passes through the cavities (maybe more if a "dogbone" configuration is used [26]) because a passive switchyard cannot separate the beam into arcs of different energies.

An FFAG, because it does not need to separate the beam into separate arcs, would seem to permit an arbitrary number of turns. However, the fact that one has no time to adjust the RF frequency limits the number of turns for which one can accelerate: one must complete acceleration before the RF phase begins to decelerate the beam. This number of turns exceeds what an RLA can accomplish only at higher energies [27, 28].

Some of the problems that must be dealt with in accelerating protons do not create difficulties for muon acceleration. Since muon energies are large compared to their mass, and since the beam emittances are extremely large, space charge effects are negligible. Also, since acceleration is extremely rapid, one passes through resonances very quickly, and therefore little emittance growth is expected due to those resonances. Thus, resonances do not lead one to desire a constant-tune FFAG.

\section{Linear Non-Scaling FFAGs}

As a result of these considerations, a linear nonscaling FFAG is a good candidate for accelerating muons. At the relativistic muon energies, a linear nonscaling FFAG can be made isochronous at an energy within its acceleration range, minimizing the time of flight variation. This allows the use of high-frequency, high-gradient RF. In particular, it allows a frequency that is compatible with the RF frequency used in muon cooling $(200 \mathrm{MHz})$. The small dispersion also reduces the horizontal magnet aperture when compared with other FFAG types, which is important for reducing the machine cost.

The largest problem facing linear non-scaling FFAGs is that the time of flight in the machine depends on the transverse amplitude of the particles. Particles with large transverse amplitudes will have different longitudinal behavior than particles with low transverse amplitudes, and 
can eventually be lost [29]. It turns out that the increase in the time of flight is proportional to the tune variation with energy [30], and inversely proportional to the energy gain per cell. To reduce the tune variation with energy, one must add nonlinear magnets, which reduce the dynamic aperture; modest amounts of nonlinearity may still give acceptable dynamic aperture. The most promising method seems to be increasing the average RF gradient; however, this can significantly reduce the number of passes one can make through the RF in the FFAG [31]. The effect would also be reduced in proportion to the emittance reduction by cooling.

There have been attempts to construct nonlinear nonscaling FFAGs for the acceleration of muons [4]. These have generally had one of two goals: to eliminate the time of flight variation with energy, or to eliminate the tune variation with energy. There are clearly benefits to either design. Unfortunately, to this point none of these designs have had sufficient dynamic aperture for muon acceleration [32]. In fact, one can demonstrate that it is not a large benefit in reducing the time of flight variation below what it naturally has for a linear nonscaling FFAG [33].

An experiment, EMMA, is being built to study linear non-scaling FFAGs [34, 35]. It will initially study rapid acceleration like that for muon acceleration. Sufficient flexibility has been built into the machine that the experiment will be able to study the dependence of the machine behavior on several different design parameters.

\section{Scaling FFAGs}

One might also consider scaling FFAGs for muon acceleration [36]. They lack tune variation with energy, so the time of flight would not depend on transverse amplitude, and the energy range would not be limited by single-cell linear resonances.

A significant disadvantage of scaling FFAGs comes from their larger magnet apertures and a larger variation of time of flight with energy when compared to linear non-scaling FFAGs. This latter effect requires lowfrequency RF (around $15 \mathrm{MHz}$ ). This RF is incompatible with the higher-frequency cooling channel, and it is also difficult to get high gradients at these low frequencies.

Instead, one could use high frequency RF with the number of RF periods changing on each turn $[9,10]$. In accelerating muons, however, one has the problem that the ring must be filled with cavities, whereas the cavities can be synchronized with the times of flight of the particles at only one position in the ring. One can adjust the frequencies of the RF cavities to maintain synchronization with the RF over a reasonable number of turns, but this only works for one direction of beam motion [37], and one would like to accelerate both signs of muons in a neutrino factory. Furthermore, the scheme is tuned for a single bunch; the RF will not be synchronized as well for other bunches in a train.

One still must deal with the large magnet apertures in the scaling FFAGs. The NuFactJ scheme, as given, had large apertures and high fields, making the machine extremely costly [38]. However, if one constructs a scaling FFAG at lower energies, one may be able to use warm or superferric magnets, which would allow wider apertures with a less significant cost penalty.

Making many passes through the cavities presents a problem of beam loading: bunches extract energy from the cavities, meaning that different bunches will see different cavity voltages. In linear non-scaling FFAGs as well as in scaling FFAG schemes at low frequency, bunches do not undergo synchrotron oscillation which would reduce the effect (there is synchrotron oscillation when using high frequency RF in a scaling FFAG). The problem is compounded by a desire to send multiple bunches from the proton driver: they must be sent within around $40 \mu \mathrm{s}$, since that is the breakup time for a mercury target [39]. There is insufficient time to re-fill the cavities, and the first train will extract a substantial amount of energy from the cavity. This will likely require the use of multiple nearby cavity frequencies in the FFAG ring.

\section{CONCLUSIONS}

FFAGs find many applications in the production of neutrino beams. They allow rapid acceleration to high energies, making them well suited for high-power proton drivers and muon acceleration. Their large energy acceptance makes them of interest in other areas of an accelerator as well.

Realizing the potential of FFAGs will require a significant continuing $R \& D$ effort in several areas:

- Cavities with high gradient that allow rapid frequency variation

- Harmonic number jump dynamics and hardware

- Space charge limits in proton FFAGs

- Optimal handling of large transverse amplitudes

- Studies of scaling FFAGs for muon acceleration

- Handling beam loading in muon FFAGs

Many of these items can be studied experimentally as well, in the EMMA and MINHA experiments.

\section{ACKNOWLEDGMENTS}

This work has been supported by US Department of Energy under contract AC02-98CH10886. 


\section{REFERENCES}

1. K. R. Symon, et al., Phys. Rev. 103, 1837-1859 (1956).

2. F. E. Mills, and C. Johnstone, in the transparency book for the $4^{\text {th }}$ International Conference on Physics Potential \& Developent of $\mu^{+} \mu^{-}$colliders, San Francisco, CA (1999), UCLA, Los Angeles, CA, pp. 693-698.

3. C. Johnstone, W. Wan, and A. Garren, "Fixed Field Circular Accelerator Design," in Proceedings of the 1999 Particle Accelerator Conference, edited by A. Luccio, and W. MacKay, IEEE, Piscataway, NJ, 1999, p. 3068.

4. G. H. Rees, Nucl. Phys. B (Proc. Suppl.) 155, 301-304 (2006).

5. J. C. Gallardo, Note on the calculation of meson $(\pi, \mathrm{K})$ production distributions for different proton bunch length, Tech. Rep. NFMCC-doc-340-v1, NFMCC (2006), URL http://nfmcc-docdb. fnal.gov/.

6. H. G. Kirk, et al., "Choice of Proton Driver Parameters for a Neutrino Factory," in [40], pp. 372-374.

7. S. Y. Lee, et al., New J. Phys. 8, 291 (2006).

8. A. G. Ruggiero, The MINHA project (2007), presentation at the FFAG 2007 workshop, Grenoble, France, April 12-17, 2007.

9. A. A. Kolomenskii, Sov. Phys. Tech. Phys. 5, 1278-1284 (1961), Russian original Zh. Tekh. Fiz. 30 (196 0), p. 1347.

10. A. G. Ruggiero, Phys. Rev. ST Accel. Beams 9, 100101 (2006).

11. Y. Mori, "PRISM: A Phase Rotator to Obtain an Intense Monochromatic Muon Beam," in Proceedings of EPAC 2000, Vienna, Austria, 2000, pp. 289-291.

12. A. Sato, et al., "R\&D Status of the High-Intense Monochromatic Low-Energy Muon Source: PRISM," in [40], pp. 2508-2510.

13. Y. Mori, Nucl. Instrum. Methods A 562, 591-595 (2006).

14. K. Okabe, M. Muto, and Y. Mori, "Development of FFAG-ERIT Ring," in [40], pp. 1675-1677.

15. C. Rubbia, et al., Nucl. Instrum. Methods A 568, 475-487 (2006).

16. D. Neuffer, Nucl. Instrum. Methods A (2007), doi:10.1016/j.nima.2007.10.047.

17. Y. Mori, "Secondary Particle Source with FFAGERIT Scheme," in The International Workshop on FFAG Accelerators, December 5-9, 2005, KURRI, Osaka, Japan, edited by Y. Mori, M. Aiba, and K. Okabe, 2006, pp. 15-20, URL http://hadron.kek.jp/FFAG/FFAG05_HP/.

18. D. Neuffer, Part. Accel. 14, 75-90 (1983).

19. D. Neuffer, "Principles and Applications of Muon Cooling," in [41], pp. 481-484.

20. V. V. Parkhomchuk, and A. N. Skrinsky, "Ionization Cooling: Physics and Applications," in [41], pp. 485-487.

21. J. C. Gallardo, et al., "New and Efficient Neutrino Factory Front-End Design," in [42], pp. 2986-2988.

22. H. Schönauer, Nucl. Instrum. Methods A 503, 318-321 (2003).

23. H. Schönauer, J. Phys. G: Nucl. Part. Phys. 29, 1739-1742 (2003).

24. A. Sato, FFAG R\&D at Osaka University, presentation at the International Workshop on FFAG Accelerators, November 6-10, 2006, KURRI, Osaka, Japan (2007).

25. J. S. Berg, et al., Phys. Rev. ST Accel. Beams 9, 011001 (2006).
26. J. S. Berg, C. Johnstone, and D. Summers, "Dogbone Geometry for Recirculating Accelerators," in [43], pp. 3323-3325.

27. J. S. Berg, and R. B. Palmer, "Cost Optimization of Non-Scaling FFAG Lattices for Muon Acceleration," in Proceedings of EPAC 2004, Lucerne, Switzerland, EPAC, European Physical Society Accelerator Group, 2004, pp. 902-904.

28. J. S. Berg, "Recent Results from Optimization Studies of Linear Non-Scaling FFAGs for Muon Acceleration,' in The International Workshop on FFAG Accelerators, October 13-16, 2004, KEK, Tsukuba, Japan, edited by S. Machida, Y. Mori, and T. Yokoi, 2005, pp. 1-8, URL http: //hadron.kek.jp/FFAG/FFAG0 4_HP/.

29. S. Machida, Phys. Rev. ST Accel. Beams 9, $10 \overline{4} 002$ (2006).

30. J. S. Berg, Nucl. Instrum. Methods A 570, 15-21 (2007).

31. J. S. Berg, "Methods for Addressing the Problem of the Dependence of Time of Flight on Transverse Amplitude in Linear Non-Scaling FFAGs," in [44], pp. 9-14, URL http: //hadron.kek.jp/FFAG/FFAG06_HP/.

32. F. Lemuet, F. Méot, and G. Rees, "6-D Beam Dynamics in an Isochronous FFAG Ring," in [42], pp. 2693-2695.

33. J. S. Berg, Optimum muon acceleration FFAG lattices without time of flight constraints, Tech. Rep. NFMCC-doc-320-v1, NFMCC (2005), URL http://nfmcc-docdb. fnal.gov/, formerly MUC-NOTE-ACCELERATION-320.

34. R. Edgecock, "EMMA-The World's First Non-Scaling FFAG," in [45], pp. 2624-2626.

35. J. S. Berg, et al., "The EMMA Lattice Design," in [45], pp. 3181-3183.

36. NuFactJ Working Group, A feasibility study of a neutrino factory in Japan (2001), URL http: //www-prism.kek.jp/nufactj/.

37. J. S. Berg, "Harmonic Number Jump in a Ring with Cavities Distributed Everywhere," in [44], pp. 69-76, URL http: //hadron.kek.jp/FFAG/FFAG06_ $\mathrm{HP} /$.

38. J. S. Berg, Status of scaling FFAG system, presentation at the 2nd International Scoping Study Meeting of the Neutrino Factory and Super-beam Facility (2006), URL http://www-kuno.phys.sci.osaka-u.ac. jp/ yoshida/ISS/index.html.

39. H. Kirk, et al., "Target Sutdies with BNL E951 at the AGS," in [43], pp. 1535-1537.

40. Proceedings of EPAC 2006, Edinburgh, Scotland, 2006.

41. F. T. Cole, and R. Donaldson, editors, Proceedings of the 12th International Conference on High-Energy Accelerators, 1983.

42. C. Horak, editor, Proceedings of 2005 Particle Accelerator Conference, Knoxville, Tennessee, IEEE, Piscataway, NJ, 2005.

43. P. Lucas, and S. Webber, editors, Proceedings of the 2001 Particle Accelerator Conference, Chicago, IEEE, Piscataway, NJ, 2001.

44. Y. Mori, K. Okabe, and T. Uesugi, editors, The International Workshop on FFAG Accelerators, November 6-10, 2006, KURRI, Osaka, Japan, 2007, URL http://hadron.kek.jp/FFAG/FFAG06_HP/.

45. C. Petit-Jean-Genaz, editor, Proceedings of PAC07, Albuquerque, New Mexico, USA, IEEE, Piscataway, NJ, 2007. 